\title{
Ubiquitous ram pressure stripping in the Coma cluster of galaxies $\star, \star \star$
}

\author{
G. Gavazzi ${ }^{1}$, G. Consolandi ${ }^{1}$, M. L. Gutierrez ${ }^{2}$, A. Boselli ${ }^{3}$, and M. Yoshida ${ }^{4}$ \\ 1 Università degli Studi di Milano-Bicocca, Piazza della Scienza 3, 20126 Milano, Italy \\ e-mail: giuseppe.gavazzi@mib.infn.it \\ 2 Instituto de Astronomia, UNAM, Km 107 Carretera Tijuana-Ensenada, 22860 Ensenada, B.C., Mexico \\ 3 Aix-Marseille Université, CNRS, CNES LAM, Laboratoire d'Astrophysique de Marseille, Marseille, France \\ 4 Subaru Telescope, National Astronomical Observatory of Japan, National Institutes of Natural Sciences, 650 A'ohoku Place, Hilo, \\ HI 96720, USA
}

Received 15 May 2018 / Accepted 16 July 2018

\begin{abstract}
We report the detection of $\mathrm{H} \alpha$ trails behind three new intermediate-mass irregular galaxies in the NW outskirts of the nearby cluster of galaxies Abell 1656 (Coma). Hints that these galaxies possess an extended component were found in earlier, deeper $\mathrm{H} \alpha$ observations carried out with the Subaru telescope. However the lack of a simultaneous $r$-band exposure, together with the presence of strong stellar ghosts in the Subaru images, prevented us from quantifying the detections. We therefore devoted one full night of $\mathrm{H} \alpha$ observation to each of the three galaxies using the San Pedro Martir $2.1 \mathrm{~m}$ telescope. One-sided tails of $\mathrm{H} \alpha$ emission of 10-20 kpc projected size were detected, suggesting an ongoing ram pressure stripping event. We added these 3 new sources of extended ionized gas to the 12 previously found, NGC 4848, and NGC 4921 whose ram pressure stripping is certified by HI asymmetry. This brings the number sources with $\mathrm{H} \alpha$ trails to 17 gaseous tails out of $27(63 \%)$ late-type galaxies (LTG) members of the Coma cluster with direct evidence of ram pressure stripping. The 27 LTG galaxies, among these the 17 with extended $\mathrm{H} \alpha$ tails, have kinematic properties that are different from the rest of the early-type galaxy population of the core of the Coma cluster, as they deviate in the phase-space diagram $|\Delta V| / \sigma$ versus $r / R_{200}$.
\end{abstract}

Key words. galaxies: evolution - galaxies: clusters: individual: Coma - galaxies: interactions - galaxies: individual: J125750.2+281013 - galaxies: individual: J125757.7+280342 - galaxies: individual: J125756.7+275930

\section{Introduction}

A $1.5 \mathrm{deg}^{2}$ region of the nearby Coma cluster of galaxies (Abell $1656,\langle z\rangle \sim 0.023,\langle c z\rangle \sim 6900 \mathrm{~km} \mathrm{~s}^{-1}$, Dist $\left.=100 \mathrm{Mpc}\right)$ was surveyed with deep $\mathrm{H} \alpha$ observations using the Subaru telescope (Yagi et al. 2010, see the inset of Fig. 5 showing the footprint of the Subaru field). These observations, carried out at the limiting surface brightness of $2.5 \times 10^{-18} \mathrm{erg} \mathrm{cm}^{-2} \mathrm{sec}^{-1} \operatorname{arcsec}^{-2}$, revealed the presence of $\mathrm{H} \alpha$ extended ionized gas (EIG) behind 14 galaxies. This indicates that, when observed with sufficiently sensitive exposures, approximately $50 \%$ of all LTGs in this cluster reveal an associated extended trail of $\mathrm{H} \alpha$.

The field of Yagi et al. (2010), however, did not cover westward of RA $12^{\mathrm{h}} 58^{\mathrm{m}} 25^{\mathrm{s}}$. For example the bright galaxy NGC 4848 was not covered by these observations and a special five hour pointing with the San Pedro Martir (SPM) $2.1 \mathrm{~m}$ telescope by Fossati et al. (2012) revealed the presence of an $\mathrm{H} \alpha$ trailing emission of $65 \mathrm{kpc}$ projected length. Similarly it did not cover the cluster eastward of RA $13^{\mathrm{h}} 01^{\mathrm{m}} 00^{\mathrm{s}}$. The bright galaxy NGC 4921 was not included, but by combining Hubble Space Telescope (HST) imaging with HI line mapping, Kenney et al. (2015) found a significant displacement of

\footnotetext{
* Based on observations taken at the San Pedro Martir telescope belonging to the Mexican National Observatory (OAN).

$\star \star$ The reduced $\mathrm{H} \alpha$ images (FITS) are only available at the CDS via anonymous ftp to cdsarc.u-strasbg.fr (130.79.128.5) or via http://cdsarc.u-strasbg.fr/viz-bin/qcat?]/A+A/618/ A130
}

the neutral hydrogen from stars; this displacement is indicative of ram pressure stripping. Another $\mathrm{H} \alpha$ Subaru pointing of the NW region of the Coma cluster was obtained in 2014 (Yagi, priv. commun.), however the absence of a corresponding broadband $(r)$ exposure to estimate and subtract the continuum emission, prevented a proper analysis of this field. However this observation hinted at possible unilateral $\mathrm{H} \alpha$ emission behind 3 additional galaxies J125750.2+281013, J125756.7+275930, and $J 125757.7+280342$ in the northwestern part of the cluster. Inspired by these tentative detections we decided to devote approximately one night of observation at the SPM observatory to each of these galaxies in April, 2018. We report the positive detection of extended, asymmetric $\mathrm{H} \alpha$ emission associated with each of these ${ }^{1}$. In total the core region of the Coma cluster covered with $\mathrm{H} \alpha$ observations contains 203 early-type galaxies (E+S0+S0a; ETGs), 27 late-type galaxies (LTGs), 17 of which show evidence of extended $\mathrm{H} \alpha$. Assuming that ionized tails arise from ram pressure stripping (Gunn \& Gott 1972) of galaxies crossing the intracluster medium (ICM) at high speed for the first time, and that the gas ablation produced by such interaction proceeds on timescales as short as $100 \mathrm{Myr}$ (Boselli \& Gavazzi 2006, 2014), we confirm that a massive infall of gas-rich, star forming galaxies (100-400 galaxies per Gyr, Adami et al. 2005; Boselli et al. 2008; Gavazzi et al. 2013a,b, 2017) is currently occurring onto rich clusters of galaxies such as Coma.

\footnotetext{
1 The three galaxies were reported as possible candidates for ram pressure stripping by Smith et al. (2010), based on GALEX selection.
} 
Table 1. Observational parameters of the target galaxies.

\begin{tabular}{lcccccccc}
\hline \hline Object & $\begin{array}{c}\text { RA } \\
(\mathrm{hh} \mathrm{m} \mathrm{s})\end{array}$ & $\begin{array}{c}\text { Dec } \\
\left({ }^{\circ} \prime^{\prime \prime}\right)\end{array}$ & $\begin{array}{c}c z \\
\mathrm{~km} \mathrm{~s}^{-1}\end{array}$ & $\begin{array}{c}\log M_{*} \\
M_{\odot}\end{array}$ & $\begin{array}{c}g-i \\
\text { mag }\end{array}$ & $\begin{array}{c}\text { H } \alpha \text { filter } \\
\AA\end{array}$ & $\begin{array}{c}\text { Texp ON } \\
\text { min }\end{array}$ & $\begin{array}{c}\text { Texp OFF } \\
\text { min }\end{array}$ \\
\hline $\mathrm{J} 125750.2+281013$ & 125750.2 & 281013 & 6936 & 8.47 & 0.22 & 6723 & 360 & 30 \\
$\mathrm{~J} 125756.7+275930$ & 125756.7 & 275930 & 4579 & 8.53 & 0.22 & 6683 & 360 & 30 \\
$\mathrm{~J} 125757.7+280342$ & 125757.7 & 280342 & 8147 & 9.75 & 0.78 & 6723 & 480 & 40 \\
\hline
\end{tabular}

Table 2. Derived $\mathrm{H} \alpha+[\mathrm{NII}]$ parameters of the target galaxies in the 3 arcsec aperture.

\begin{tabular}{lcccccc}
\hline \hline Object & $\begin{array}{c}\text { EW 3 } \\
\AA\end{array}$ & $\begin{array}{c} \pm \\
\AA\end{array}$ & $\begin{array}{c}\text { Log Flux 3" } \\
\mathrm{erg} \mathrm{cm}^{-2} \mathrm{sec}^{-1}\end{array}$ & $\begin{array}{c} \pm \\
\mathrm{erg} \mathrm{cm}^{-2} \mathrm{sec}^{-1}\end{array}$ & $\begin{array}{c}\text { EW SDSS } \\
\AA\end{array}$ & $\begin{array}{c}\text { Log flux SDSS } \\
\mathrm{erg} \mathrm{cm}^{-2} \mathrm{sec}^{-1}\end{array}$ \\
\hline $\mathrm{J} 125750.2+281013$ & -25.8 & 3.4 & -14.75 & 0.05 & -36.6 & -14.68 \\
$\mathrm{~J} 125756.7+275930$ & -90.4 & 4.7 & -14.25 & 0.02 & -100.8 & -14.24 \\
$\mathrm{~J} 125757.7+280342$ & -63.2 & 4.2 & -14.04 & 0.02 & -45.9 & -14.14 \\
\hline
\end{tabular}

Table 3. Derived total $\mathrm{H} \alpha+[\mathrm{NII}]$ parameters of the target galaxies.

\begin{tabular}{lcccccc}
\hline \hline Object & $\begin{array}{c}\text { EW tot } \\
\AA\end{array}$ & $\begin{array}{c} \pm \\
\AA\end{array}$ & $\begin{array}{c}\text { Log flux tot } \\
\mathrm{erg} \mathrm{cm}^{-2} \mathrm{sec}^{-1}\end{array}$ & $\begin{array}{c} \pm \\
\mathrm{erg} \mathrm{cm}^{-2} \mathrm{sec}^{-1}\end{array}$ & $\begin{array}{c}\text { Limiting } \Sigma \\
\mathrm{erg} \mathrm{cm}^{-2} \mathrm{sec}^{-1}\end{array}$ & $\begin{array}{c}\text { Proj. length } \\
\mathrm{kpc}\end{array}$ \\
\hline $\mathrm{J} 125750.2+281013$ & -13.7 & 3.1 & -14.01 & 0.09 & $5.88 \times 10^{-18}$ & 10 \\
$\mathrm{~J} 125756.7+275930$ & -56.3 & 3.7 & -13.47 & 0.02 & $5.88 \times 10^{-18}$ & 22 \\
$\mathrm{~J} 125757.7+280342$ & -20.3 & 3.4 & -13.28 & 0.07 & $5.01 \times 10^{-18}$ & 24 \\
\hline
\end{tabular}

\section{Observations}

On the nights of April 12, 13, and 14, 2018, we used the $2.1 \mathrm{~m}$ telescope at SPM to repeatedly observe three $5 \times$ $5 \operatorname{arcmin}^{2}$ regions of the nearby Coma cluster of galaxies, each targeting one of the J125750.2+281013, J125756.7+275930, $\mathrm{J} 125757.7+280342$ galaxies. We used two narrowband $(80 \AA)$ filters centered at $\lambda 6683$ and $\lambda 6723 \AA$ to detect the $\mathrm{H} \alpha$ emission (at the redshift listed in Table 1 along with other parameters taken from GOLDmine; Gavazzi et al. 2003) and a broadband $r$ (Gunn) filter (effective $\lambda 6231 \AA, \Delta \lambda \sim 1200 \AA$ ) to recover the continuum emission.

The fields were observed with several individual pointings of $1800 \mathrm{~s}$ in the ON-band filter, and with same number of $300 \mathrm{~s}$ exposures with the broadband (OFF-band) $r$ filter, up to a total exposure time given in Table 1 . After flat fielding, the aligned observations were combined into a final ON-band frame and an OFF-band frame (details on the reduction procedures of $\mathrm{H} \alpha$ observations can be found in Gavazzi et al. 2012).

To calibrate the data, we repeatedly observed the spectrophotometric stars Feige34 and Hz44 (Massey et al. 1988). From the calibrated data we extracted the flux and the EW within a circular aperture of 3 arcsec (diameter) and we checked the calibration comparing these values with the measurements of $\mathrm{H} \alpha+[\mathrm{NII}]$ flux and EW extracted from the Sloan Digital Sky Survey (SDSS) nuclear fiber spectra (see Table 2). In spite of the uncertainty in the absolute positioning of the central aperture, the agreement between two sets of data is satisfactory, reassuring us concerning the quality of the photometric calibration. The integrated photometric parameters are listed in Table 3. Among these the projected length gives the approximate full extension of the $\mathrm{H} \alpha$ tail from the center of the parent galaxy.

In Table 1 we list the celestial coordinates, redshift, total stellar mass, derived from the $i$-band luminosity and the $g-i$ color according to Zibetti et al. (2009), assuming a Chabrier initial mass function (IMF; Chabrier 2003). Our observations, owing to the generous integration time of $6 / 8 \mathrm{~h}$ (ON band), reached a deep sensitivity limit and provided the estimate of other extended parameters, as listed in Table 3.

\section{Estimate of ionized gas mass}

An estimate of the density of the ionized gas in the tail of $\mathrm{J} 125756.7+275930$, the most clear cut candidate for ram pressure stripping, can be achieved using Eqs. (1) and (2) in Boselli et al. (2016a). The stripped material is assumed to be distributed in a cylinder of diameter of $5 \mathrm{kpc}$ and of length comparable to the deprojected extension of the tail of ionized gas. Since the galaxy has a redshift of $4579 \mathrm{~km} \mathrm{~s}^{-1}$ (as compared to the mean cluster velocity of $6900 \mathrm{~km} \mathrm{~s}^{-1}$ ), it is crossing the cluster from behind. Thus the real length of the tail can be roughly estimated multiplying the observed length by $\sqrt{3}$, obtaining $38 \mathrm{kpc}$. The region containing the stripped gas (assumed with a filling factor of 0.1 ) has a volume of $710 \mathrm{kpc}^{3}$ and a luminosity $L(\mathrm{H} \alpha)$ of $2.44 \times 10^{40} \mathrm{erg} \mathrm{sec}^{-1}$, assuming [NII]/H $\alpha=0.1$ and $70 \%$ of the total $\mathrm{H} \alpha$ emission in the tail. Thus the region has a density of electrons (or protons) of $0.19 \mathrm{~cm}^{-3}$, which translates into a total mass of $3.05 \times 10^{8} M_{\odot}$ of ionized gas. This is consistent with $3.55 \times 10^{8} M_{\odot}$ MHI lost in the tail, roughly estimated from the measured $\mathrm{MHI}=10^{8.62} M_{\odot}$, combined with a deficiency parameter of $\operatorname{Def}_{\mathrm{HI}}=0.27$.

\section{Discussion}

The full $\mathrm{H} \alpha$ survey of the central region of the Coma cluster (covering approximately $12^{\mathrm{h}} 57^{\mathrm{m}}-13^{\mathrm{h}} 02^{\mathrm{m}} ;+27^{\mathrm{d}} 30^{\mathrm{p}}-28^{\mathrm{d}} 17^{\mathrm{p}}$ ), including the work of Yagi et al. (2010), was extended to the NW by Fossati et al. (2012) and by the present work. Assuming the $r=17.7$ mag threshold adopted by SDSS, this sky window 
Table 4. Properties of the 27 surveyed LTG galaxies (plus 2 ETG with tails).

\begin{tabular}{|c|c|c|c|c|c|c|c|c|c|c|c|}
\hline Jname & GMP & CGCG & NGC & Other & $\begin{array}{c}c z \\
\mathrm{~km} \mathrm{~s}^{-1}\end{array}$ & Type & $\begin{array}{c}r \\
\text { mag }\end{array}$ & $\begin{array}{l}g-i \\
\text { mag }\end{array}$ & $\begin{array}{c}\log M_{*} \\
M_{\odot} \\
\end{array}$ & $\operatorname{Def}_{\mathrm{HI}}$ & Ref $\mathrm{H} \alpha$ \\
\hline SDSSJ125750.2+281013 & & & & & 6935 & $\mathrm{BCD}$ & 17.14 & 0.20 & 8.47 & - & T.W. \\
\hline SDSSJ125756.7+275930 & & & & & 4579 & PEC & 16.94 & 0.18 & 8.53 & 0.27 & T.W. \\
\hline SDSSJ125757.7+280342 & & & & & 8147 & PEC & 15.24 & 0.70 & 9.75 & 0.53 & T.W. \\
\hline SDSSJ125805.6+281433 & & 160055 & 4848 & & 7193 & $\mathrm{Sab}$ & 13.83 & 0.88 & 10.54 & 0.25 & Fossati+12 \\
\hline SDSSJ125818.2+275054 & & & & & 7649 & $\mathrm{BCD}$ & 17.05 & 0.41 & 8.69 & - & \\
\hline SDSSJ125819.7+280541 & & & & & 7197 & $\mathrm{Sm}$ & 17.19 & 0.41 & 8.58 & - & \\
\hline SDSSJ125825.5+280744 & & & & & 8212 & PEC & 16.23 & 0.62 & 9.23 & - & \\
\hline SDSSJ125830.7+273352 & 4232 & & & & 7285 & S.. & 17.75 & 0.41 & 7.39 & - & Yagi+10 \\
\hline SDSSJ125838.1+274921 & & & & & 5424 & $\mathrm{Sm}$ & 17.35 & 0.56 & 8.67 & - & \\
\hline SDSSJ125842.5+274537 & 4060 & & & & 8753 & $\mathrm{Sm}$ & 16.78 & 0.40 & 8.80 & - & Yagi+10 \\
\hline SDSSJ125856.0+275000 & 3896 & 160212 & & IC3949 & 7553 & $\mathrm{Sa}$ & 14.20 & 0.71 & 10.27 & 1.01 & Yagi+10 \\
\hline SDSSJ125902.0+280656 & 3816 & 160213 & 4858 & & 9430 & $\mathrm{Sb}$ & 15.21 & 0.62 & 9.70 & 0.96 & Yagi+10 \\
\hline SDSSJ125905.2+273840 & 3779 & 160073 & & & 5435 & $\mathrm{Sa}$ & 14.37 & 0.56 & 10.00 & 0.82 & Yagi +10 \\
\hline SDSSJ125914.9+281503 & & & & & 7499 & $\mathrm{Sm}$ & 16.09 & 0.36 & 9.11 & - & \\
\hline SDSSJ125939.8+273435 & 3271 & & & & 5007 & PEC & 15.75 & 0.50 & 9.34 & - & Yagi+10 \\
\hline SDSSJ125941.3+273935 & & & & & 6748 & $\mathrm{Sa}$ & 15.10 & 1.07 & 10.07 & - & \\
\hline SDSSJ125956.1+274446 & 3071 & & & & 8975 & $\mathrm{BCD}$ & 16.21 & 0.37 & 9.05 & - & Yagi+10 \\
\hline SDSSJ130001.0+280455 & 3016 & & & & 7770 & S.. & 17.8 & 0.48 & 7.49 & - & Yagi +10 \\
\hline SDSSJ130008.0+274623 & 2923 & & & & 8726 & $\mathrm{BCD}$ & 16.89 & 0.37 & 8.73 & - & Yagi +10 \\
\hline SDSSJ130009.7+275158 & 2910 & 160243 & & D100 & 5316 & $\mathrm{BCD}$ & 15.47 & 0.61 & 9.56 & - & Yagi+10 \\
\hline SDSSJ130013.4+280311 & & & & & 8205 & $\mathrm{~S} . .$. & 17.61 & 0.69 & 8.65 & - & \\
\hline SDSSJ130033.7+273815 & & 160086 & & & 7497 & $\mathrm{Sb}$ & 15.30 & 0.39 & 9.47 & 0.59 & \\
\hline SDSSJ130037.9+280326 & 2559 & 160252 & & IC4040 & 7637 & $\mathrm{Sdm}$ & 15.07 & 0.60 & 9.78 & 0.45 & Yagi+10 \\
\hline SDSSJ130045.2+274449 & & & & & 9361 & $\mathrm{Sd}$ & 17.55 & 0.66 & 8.64 & - & \\
\hline SDSSJ130056.0+274726 & 2374 & 160260 & 4911 & & 7995 & $\mathrm{Sa}$ & 13.03 & 1.18 & 11.11 & 0.31 & Yagi+10 \\
\hline SDSSJ130119.3+275137 & & & & & 8260 & $\mathrm{BCD}$ & 17.02 & 0.63 & 8.86 & - & \\
\hline SDSSJ130126.1+275309 & & 160095 & 4921 & & 5481 & $\mathrm{Sb}$ & 12.90 & 1.22 & 11.20 & 0.73 & Kenney +15 \\
\hline SDSSJ125847.4+274029 & 4017 & 160070 & 4854 & & 8400 & S0 & 13.86 & 1.22 & 10.74 & - & Yagi+10 \\
\hline SDSSJ125835.2+273547 & 4156 & 160068 & 4853 & & 7710 & So & 13.51 & 1.03 & 10.78 & - & Yagi +10 \\
\hline
\end{tabular}

contains 203 ETGs (E/S0/S0a) and 27 LTGs (Sa-Sm-BCD) (as listed in Table 4, taken from GOLDMine). Among the LTGs, 12 are found associated with cometary $\mathrm{H} \alpha$ tails from Yagi et al. $(2010)^{2}$. One (N4848) was found extended by Fossati et al. (2012), and 3 in this work. In addition, NGC 4921, while not showing extended $\mathrm{H} \alpha$ emission, was considered an example of galaxy subject to ram pressure stripping because of its asymmetric HI distribution by Kenney et al. (2015). In total there are $17 / 27$ (63\%) LTGs (plus 2 ETGs) with $\mathrm{H} \alpha$ cometary tails or HI asymmetry indicating ram pressure. In conclusion more than one out of two LTGs, possibly gas rich systems in this cluster, show cometary $\mathrm{H} \alpha$ debris, which is a signature of ram pressure stripping. This reinforces a previous result for A1367 where 11/26 or $42 \%$ of the surveyed LTGs show $\mathrm{H} \alpha$ tails (Consolandi et al. 2017).

Some properties of the 27 surveyed LTG galaxies (+2 ETG with tails) are listed in Table 4. In particular we list the $r$ magnitude and the $g-i$ color (SDSS, as given by GOLDMine) and the HI deficiency parameter. This parameter was defined by Haynes \& Giovanelli (1984) as $\operatorname{Def}_{\mathrm{HI}}=$ $\langle\log \mathrm{MHI}(T, \mathrm{~d} L)\rangle-\log \mathrm{MHI}(\mathrm{obs})$ where $\langle\log \mathrm{MHI}(T, \mathrm{~d} L)\rangle=\mathrm{C} 1+$ $\mathrm{C} 2 \times 2 \log (\mathrm{d} L)$, where $\mathrm{d} L[\mathrm{kpc}]$ is determined in the $g$-band at the 25 th mag arcsec ${ }^{-2}$ isophote. References to the HI measurements can be found in Gavazzi et al. (2006), however the deficiency

\footnotetext{
2 The full number of $\mathrm{H} \alpha$ tails in Yagi et al. (2010) is 14, but two are associated with ETGs, as listed in the last lines of Table 4.
}

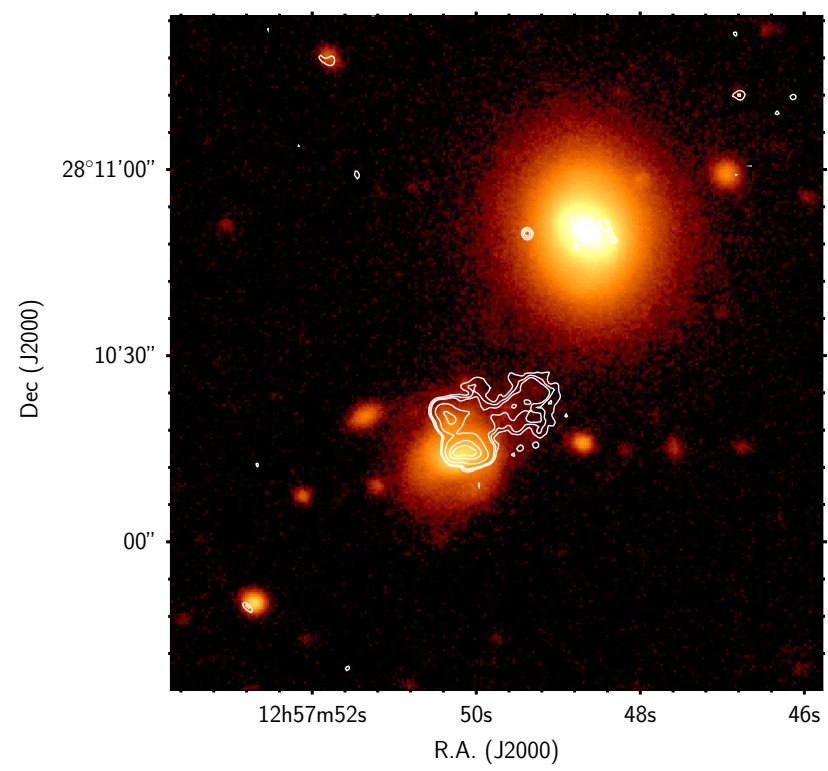

Fig. 1. Grayscale representation of the $r$-band emission from J125750.2+281013. Contours represent the $\mathrm{H} \alpha$ NET emission. The proximity of the massive galaxy CGCG 160-049 does not allow us to exclude that the gaseous asymmetry is due to tidal interaction. However we note that stars appear not accordingly displaced. Levels are -17.07 $-16.89-16.29-15.87-15.69 \mathrm{erg} \mathrm{cm}^{-2} \mathrm{sec}^{-1} \operatorname{arcsec}^{-2}$ in log units, after three pixel Gaussian smoothing. 


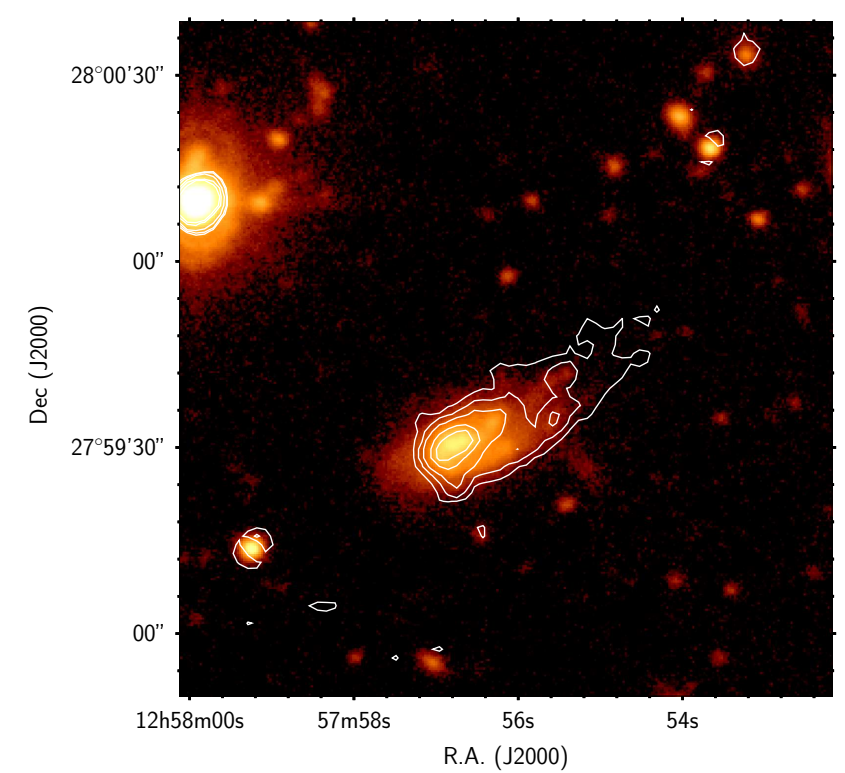

Fig. 2. Same as Fig. 1 for J125756.7+275930. Levels are -17.30 - 17.07 $-16.77-15.77-15.47 \mathrm{erg} \mathrm{cm}^{-2} \mathrm{sec}^{-1} \mathrm{arcsec}^{-2}$ in log units, after three pixel Gaussian smoothing.

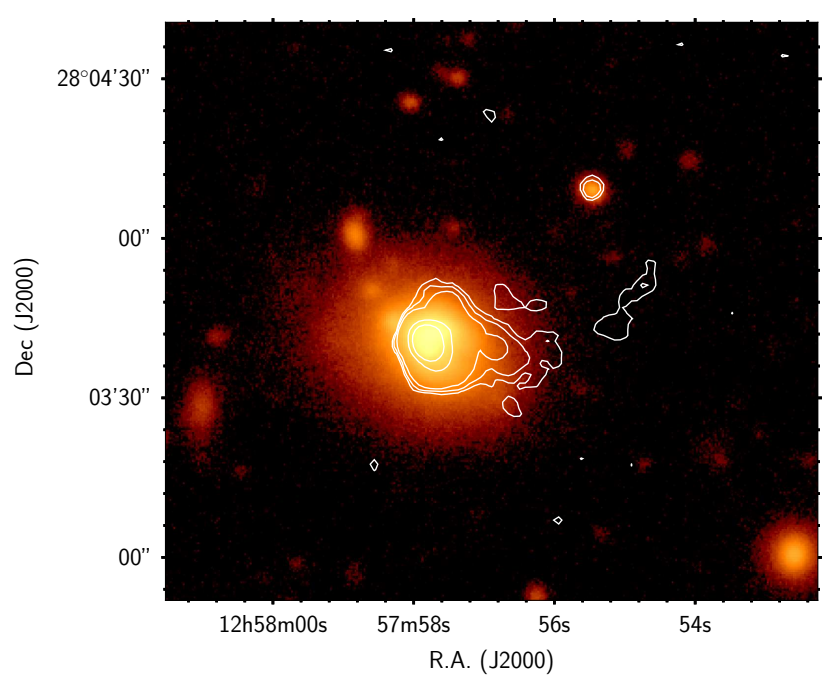

Fig. 3. Same as Fig. 1 for J125757.7+280342. Levels are $-17.30-16.77$ $-16.30-15.77-15.47 \mathrm{erg} \mathrm{cm}^{-2} \mathrm{sec}^{-1} \operatorname{arcsec}^{-2}$ in log units, after three pixel Gaussian smoothing.

parameter is recomputed in this work adopting $\mathrm{C} 1=7.51$ and C2 $=0.68$ for all LTGs (Sa-BCD) (as discussed by Gavazzi et al. 2013a). The HI deficiency parameter is unfortunately available only for 10/27 galaxies. It is noticeable that all available values are found between 0.25 and 1.01, implying an HI deficiency factor between 1.8 and 10 times the normal HI content of LTGs.

The spatial distribution and pointing direction of the $\mathrm{H} \alpha$ tails detected by Yagi et al. (2010) was sketched in their work (see their Fig. 6). To further stress the contribution of the present work a smaller area of the cluster is sketched in Fig. 6 where the distribution of galaxies, as derived from the SDSS is given in grayscale. Superposed are the X-ray contours of the hot gas emission (as revealed by XMM) embedding the galaxies. The head-tail radio galaxy 5C 04.081 is represented in red contours. Blue contours give the $\mathrm{H} \alpha$ extended emission from either Yagi et al. (2010), Fossati et al. (2012), or the present work. All

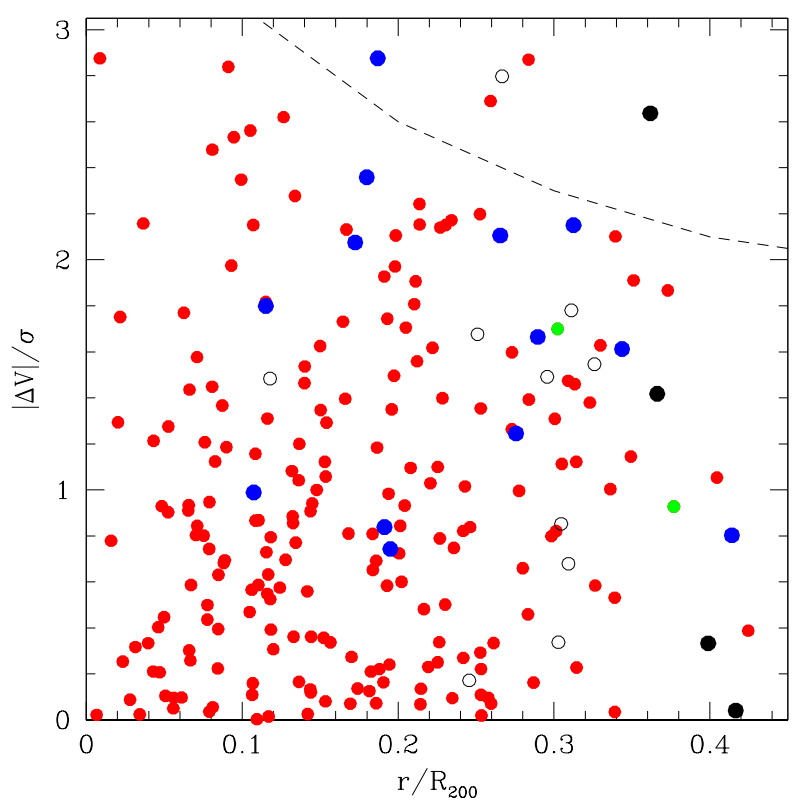

Fig. 4. Phase-space diagram of the core of the Coma cluster surveyed in $\mathrm{H} \alpha .200$ Elliptical+S0 galaxies are indicated in red (except two with $\mathrm{H} \alpha$ tails in green), 10 LTGs without $\mathrm{H} \alpha$ extension are given with open symbols, and 17 LTGs with $\mathrm{H} \alpha$ tails are over plotted in blue, except NGC 4848 and the 3 galaxies analyzed in this paper shown with filled black symbols. The dashed line represents the escape velocity in a Comalike cluster. We adopt for Coma $R_{200}=2.19 \mathrm{Mpc}$ and $\sigma=880 \mathrm{~km} \mathrm{~s}^{-1}$ (Boselli \& Gavazzi 2006).

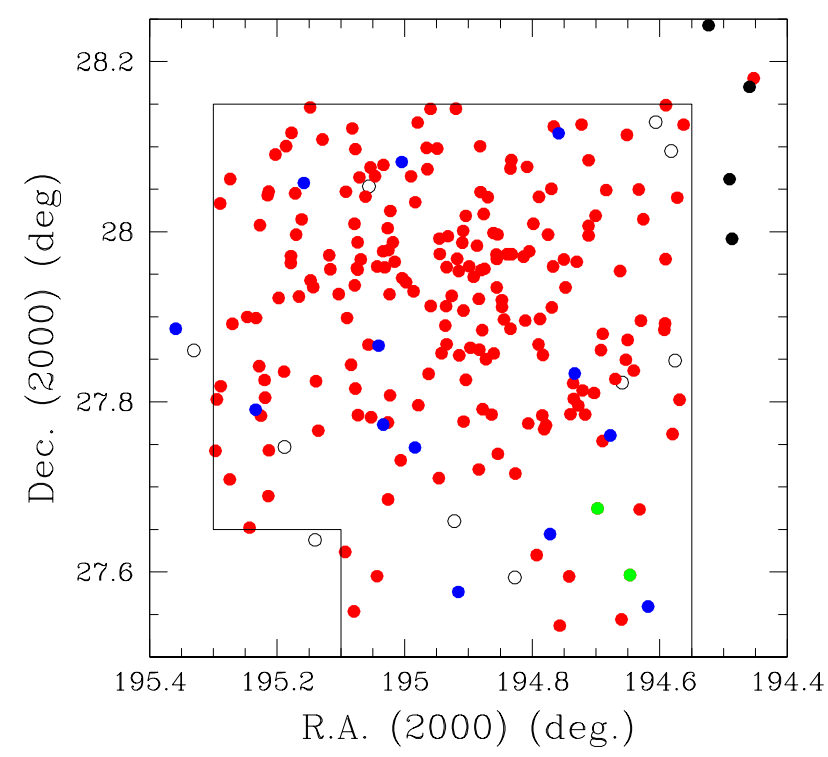

Fig. 5. Sky distribution of the core of the Coma cluster surveyed in $\mathrm{H} \alpha$ by (Yagi et al. 2010; region within black lines), plus the 4 galaxies observed in this work or by Fossati et al. (2012; in the NW shown in filled black) and NGC4921 (Kenney et al. 2015 in the far E). Same symbols as in Fig. 4.

tails lying in the NW part of the cluster, including NGC 4848, point toward NW, indicating that the prevailing infall direction is from NW to SE. The third dimension (along the line of sight) is however unclear: out of the four galaxies in this part of the cluster, two have almost no velocity difference with respect to the cluster (suggesting motion in the plane of the sky), the other two have opposite relative line-of sight veloci- 


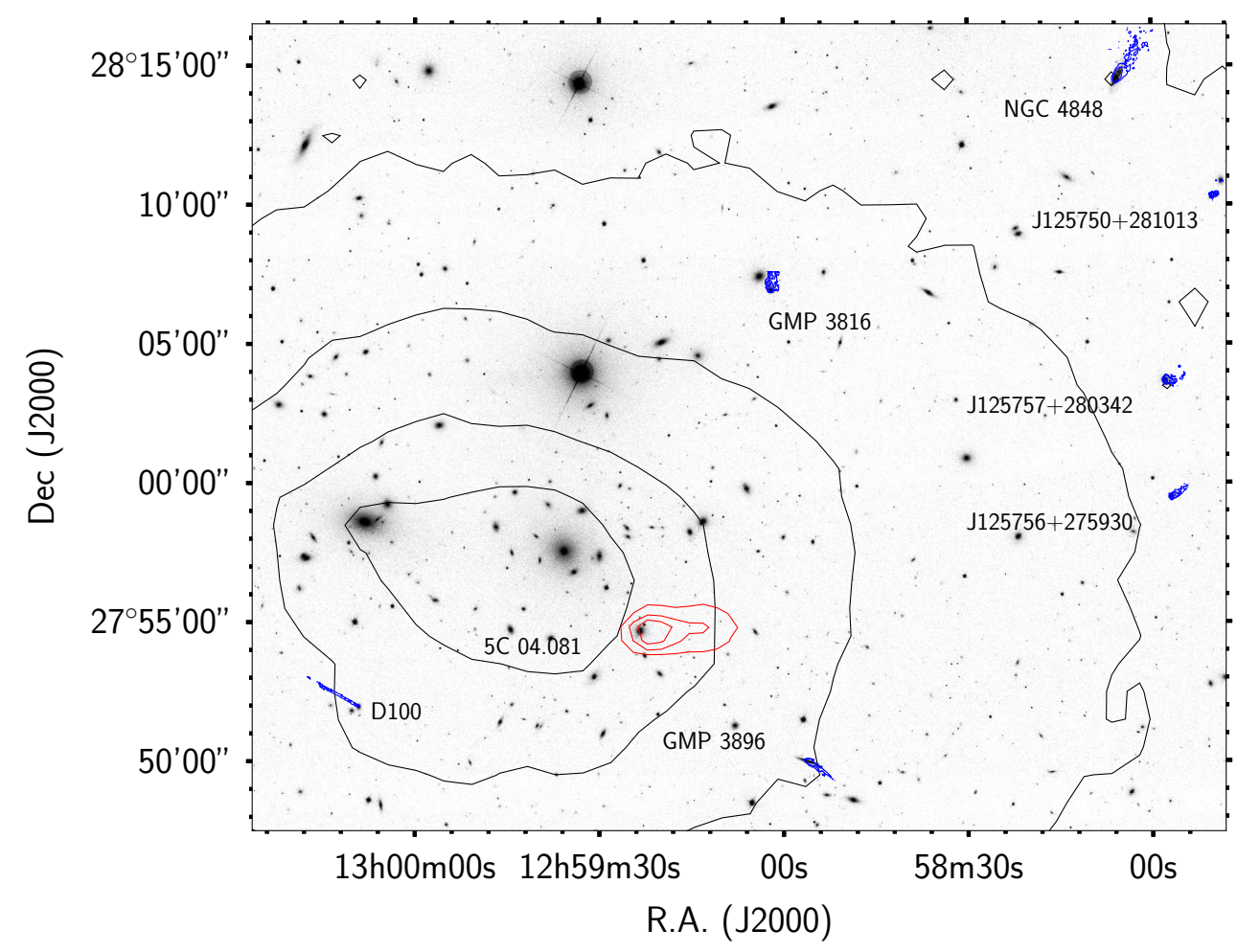

Fig. 6. Grayscale representation of the distribution of galaxies in the NW part of the Coma cluster (from SDSS) to highlight the position of the newly discovered $\mathrm{H} \alpha$ tails. Black contours represent the X-ray emission (XMM 0.4$1.3 \mathrm{keV}$, contour levels: 10, 20, 50, 100,150 cnts per second per square degree with a smoothing of 10 pixel). Red contours represent the radio emission from the head-tail radio source 5C 04.081. Blue contours show the $\mathrm{H} \alpha$ NET emission for NGC 4848 from this work, Yagi et al. (2010), and Fossati et al. (2012). ties $\left(-2321,+1247 \mathrm{~km} \mathrm{~s}^{-1}\right)$, which do not indicate a common provenance.

We quantify in Fig. 4 the kinematical difference of the virialized galaxies (ETGs) from all LTGs (probably infalling into the cluster) and from LTGs that are currently losing their gas from ram pressure, as shown by the appearance of unilateral gaseous tails. This is shown with the phase-space test of Boselli et al. (2014) and Jaffé et al. (2015) on the core region of the Coma cluster surveyed in $\mathrm{H} \alpha$. Considering all SDSS galaxies with $r$ brighter than $17.7 \mathrm{mag}$, this region contains 203 ETGs (E/S0/S0a, of which two with extended $\mathrm{H} \alpha$ tails), 27 LTGs, of which 17 display an $\mathrm{H} \alpha$ cometary tail. It is self evident that LTGs in general, and in particular tailed LTGs, differ from the distribution of ETGs, i.e., at any radius the LTGs (and even more the tailed LTGs) show higher peculiar normalized velocities, closer to the cluster escape velocity. Disregarding the radial dependence of $|\Delta V| / \sigma$, we derive overall $\langle|\Delta V| / \sigma\rangle=0.94$ for ETGs, 1.53 and 1.28 for LTGs with and without $\mathrm{H} \alpha$ tails, respectively. We must stress, however, that at large $r / R_{200} \sim 0.4$, where two new NW LTG tails and NGC 4848 are found, their normalized velocity is $\leq 0.4$, which is much below the escape velocity; this is because they were not in infall, or their infall was in the plane of the sky. Finally, we note that the two ETGs, albeit empty from star formation at their interior, show extended ionized gas (see the last two lines of Table 4). It is possible that their gas was stripped in a previous (preprocessing) phase. We conclude by speculating that our analysis is consistent with the idea that all LTGs are currently infalling, but $40 \%$ of them are not seen with extended cometary tails because they have not actually entered the dense intergalactic medium (IGM) of the cluster, in spite of being projected on it, or because they have been totally stripped in the past. The conspicuous infall rate that we observe in Coma and A1367 give us hope that a similar, or even higher rate exists in the Virgo cluster, and will be revealed by the foreseen Virgo Environmental
Survey Tracing Ionised Gas Emission (VESTIGE) announced by Boselli et al. (2018).

Acknowledgements. This research has made use of the GOLDmine database (Gavazzi et al. 2003, 2014) and of the NASA/IPAC Extragalactic Database (NED), which is operated by the Jet Propulsion Laboratory, California Institute of Technology, under contract with the National Aeronautics and Space Administration. We thank the anonymous referee for his/her constructive criticism.

\section{References}

Adami, C., Biviano, A., Durret, F., \& Mazure, A. 2005, A\&A, 443, 17 Boselli, A., \& Gavazzi, G. 2006, PASP, 118, 517

Boselli, A., \& Gavazzi, G. 2014, A\&ARv, 22, 74

Boselli, A., Boissier, S., Cortese, L., \& Gavazzi, G. 2008, ApJ, 674, 742 Boselli, A., Voyer, E., Boissier, S., et al. 2014, A\&A, 570, A69

Boselli, A., Cuillandre, J. C., Fossati, M., et al. 2016a, A\&A, 587, A68 Boselli, A., Roehlly, Y., Fossati, M., et al. 2016b, A\&A, 596, A11 Boselli, A., Fossati, M., Ferrarese, L., et al. 2018, A\&A, 614, A56 Chabrier, G. 2003, PASP, 115, 763

Consolandi, G., Gavazzi, G., Fossati, M., et al. 2017, A\&A, 606, A83 Fossati, M., Gavazzi, G., Boselli, A., \& Fumagalli, M. 2012, A\&A, 544, A128

Fossati, M., Fumagalli, M., Boselli, A., et al. 2016, MNRAS, 455, 2028 Gavazzi, G., Boselli, A., Donati, A., Franzetti, P., \& Scodeggio, M. 2003, A\&A, 400,451

Gavazzi, G., O’Neil, K., Boselli, A., \& van Driel, W. 2006, A\&A, 449, 929 Gavazzi, G., Fumagalli, M., Galardo, V., et al. 2012, A\&A, 545, A16 Gavazzi, G., Fumagalli, M., Fossati, M., et al. 2013a, A\&A, 553, A89 Gavazzi, G., Savorgnan, G., Fossati, M., et al. 2013b, A\&A, 553, A90 Gavazzi, G., Franzetti, P., \& Boselli, A. 2014, ArXiv e-prints [arXiv:1401.8123] Gavazzi, G., Consolandi, G., Yagi, M., \& Yoshida, M. 2017, A\&A, 606, A131 Gunn, J. E., \& Gott, J. R., III 1972, ApJ, 176, 1

Haynes, M. P., \& Giovanelli, R. 1984, AJ, 89, 758

Jaffé, Y. L., Smith, R., Candlish, G. N., et al. 2015, MNRAS, 448, 1715 Kenney, J. D. P., Abramson, A., \& Bravo-Alfaro, H. 2015, AJ, 150, 59 Massey, P., Strobel, K., Barnes, J. V., \& Anderson, E. 1988, ApJ, 328, 315 Smith, R. J., Lucey, J. R., Hammer, D., et al. 2010, MNRAS, 408, 141 Yagi, M., Yoshida, M., Komiyama, Y., et al. 2010, AJ, 140, 1814 Yagi, M., Yoshida, M., Gavazzi, G., et al. 2017, ApJ, 839, 65 Zibetti, S., Charlot, S., \& Rix, H.-W. 2009, MNRAS, 400, 1181 